\title{
Assessing the pathogenicity of MLH1 missense mutations in patients with suspected hereditary nonpolyposis colorectal cancer: correlation with clinical, genetic and functional features
}

\author{
Laura Belvederesi ${ }^{1,2,5}$, Francesca Bianchi ${ }^{1,2,5}$, Cristian Loretelli ${ }^{1,2}$, Daniela Gagliardini ${ }^{1,2}$, \\ Eva Galizia $^{1,2}$, Raffaella Bracci ${ }^{1,2}$, Saverio Rosati ${ }^{1,2}$, Italo Bearzi ${ }^{3}$, Alessandra Viel ${ }^{4}$, \\ Riccardo Cellerino ${ }^{1,2}$ and Emilio Porfiri*,1
}

\footnotetext{
${ }^{1}$ Oncologia Medica, Facolta' di Medicina e Chirurgia, Università Politecnica delle Marche, Ancona, Italy; ${ }^{2}$ Centro Regionale di Genetica Oncologica, Ancona, Italy; ${ }^{3}$ Anatomia ed Istologia Patologica, Facolta' di Medicina e Chirurgia, Università Politecnica delle Marche, Ancona, Italy; ${ }^{4}$ Oncologia Sperimentale 1, Dipartimento di Ricerca Preclinica ed Epidemiologica, Centro Riferimento Oncologico, IRCCS, Aviano, Italy
}

Assessing the pathogenicity of missense mutations of $M L H 1$ and $M S H 2$ is critical to counsel patients with suspected hereditary nonpolyposis colorectal cancer (HNPCC). Approximately $32 \%$ of all MLH1 mutations and $18 \%$ of $M S H 2$ mutations are missense variants which often have an uncertain genetic significance. To assess the pathogenicity of four MLH1 missense mutations which were found in five patients with suspected HNPCC, P648S (CCC $\rightarrow$ TCC), L559R (CTG $\rightarrow$ CGG), K618A (AAG $\rightarrow$ GCG), Y646C (TAT $\rightarrow$ TGT), we studied their ability to disrupt MLH1 protein function and their relationship with all those clinical, genetic and pathological features which are typical of this syndrome. Our results indicated that the P648S and L559R mutations were probably pathogenic because they disrupted MLH1 protein interaction with its partner PMS2 in vitro and abolished MLH1 expression in HCT116 cells. In addition these variants were associated with features often found in HNPCC patients: in particular high microsatellite instability, occurrence of high grade tumours and, in one case, strong family history. The pathogenicity of the K618A and Y646C mutations was questionable as their correlation with features typical of HNPCC was low and the outcome of the functional analysis was ambiguous. These observations suggested that a clinically usable assessment of the pathogenicity of $M L H$ missense variants can be achieved through the analysis of multiple mutation characteristics among which loss of protein function, occurrence of microsatellite instability and family history seemed to have a predominant role.

European Journal of Human Genetics (2006) 14, 853-859. doi:10.1038/sj.ejhg.5201628; published online 17 May 2006

Keywords: $M L H 1$; missense mutation; HNPCC

${ }^{*}$ Correspondence: Dr E Porfiri, Cancer Research - UK Institute for Cancer Studies, University of Birmingham, Vincent Drive, Birmingham B15 2TT, UK. Tel: + 440121414 2808; Fax: + 440121414 4486;

E-mail: e.porfiri@bham.ac.uk

${ }^{5}$ These authors contributed equally to this work

Received 8 September 2005; revised 27 February 2006; accepted 28 February 2006; published online 17 May 2006
Introduction

Hereditary nonpolyposis colorectal cancer (HNPCC) is a syndrome of cancer predisposition linked to inherited mutations of genes participating in postreplicative DNA mismatch repair (MMR). ${ }^{1}$ The products of several genes, including MLH1, MSH2, MSH6, PMS2, MLH3, MSH3 and PMS1, participate in this process nevertheless approximately $90 \%$ of the germline mutations found in HNPCC 
families involve MLH1 and MSH2 whereas mutations of PMS2, MSH6, MLH3 and PMS1 have been found in fewer cases. ${ }^{1-3}$ The MSH2 protein, by interacting with MSH6 or MSH3, regulates mismatch recognition. ${ }^{3,4}$ The MLH1 protein, by forming the MutL $\alpha$ complex with PMS 2 or by binding to MLH3 or PMS1, coordinates the interaction between the mismatch recognition machinery and the other proteins participating in DNA repair. ${ }^{3,5}$ Inactivation of the MMR system results in the accumulation of DNA replication errors which are thought to contribute to tumour development and are responsible for microsatellite instability (MSI), a common genetic feature of HNPCC tumours. ${ }^{3}$ So far more than 450 germline abnormalities of MMR genes have been described and are listed in the database of the International Society for Gastrointestinal Hereditary Tumours (InSiGHT, http://www.insight-group.org). The majority of these mutations are easily recognized as pathogenic as they result in the expression of truncated proteins. However, $32 \%$ of MLH1 mutations and $18 \%$ of MSH2 mutations are missense variants which cause single amino-acid substitutions and often have a dubious pathogenicity. $^{2}$

Several criteria have been used to assess the pathogenicity of missense mutations: cosegregation with the disease, low incidence in the general population, substitution of evolutionary conserved amino acids, nonconservative amino-acid changes and, in the case of suspected HNPCC, correlation with MSI and loss of MMR gene expression. ${ }^{6,7}$ Unfortunately these criteria are frequently insufficient to predict the pathogenicity of missense variants: segregation studies are rarely feasible particularly in small families or when clinical specimens are not available, ${ }^{6}$ nonconservative amino-acid changes may also be caused by polymorphisms, ${ }^{6,8}$ in addition loss of MMR gene expression and MSI could represent the result of somatic mutations or epigenetic abnormalities such as promoter methylation. ${ }^{9}$ In order to estimate the pathogenicity of MLH1 missense mutations several functional assays have been developed. These assays have shown that certain mutations result in a decreased ability of MLH1 to interact with PMS2 $2^{10,11}$ or in loss of DNA MMR functions. ${ }^{8,12,13}$ Although these studies have generated valuable observations, it is difficult to establish their clinical impact because different assays have been used, and because the results of the MMR tests are often analysed with few references to the clinical context in which the mutation was originally detected.

The identification of subjects carrying genetic abnormalities linked to HNPCC has broad clinical and psychological implications due to the greatly increased cancer risk of these individuals. ${ }^{14}$ A clinically usable protocol to assess the pathogenicity of missense variants is urgently warranted, such protocol to be reliable should take into account the clinical, genetic and functional characteristics of the mutation. Here, we describe the approach we used to assess the pathogenicity of four MLH1 missense variants which were found in five patients with suspected HNPCC. In the first instance we analysed the relationship of the four mutations with the familial cancer history of the patients, with the occurrence of MSI and with the abnormalities of MMR gene expression in the tumour tissue. In addition we used multiplex ligation-dependent probe amplification (MLPA) to exclude the occurrence of large genomic deletions of MLH1 and MSH2. We then studied the frequency of the mutations in a panel of healthy controls, the type of amino-acid change and whether the mutated amino-acid residue was evolutionally conserved. Finally, we correlated these features with the ability of each mutation to disrupt the interaction of the MLH1 protein with PMS2 in vitro and to affect the expression of MLH1 in colon cancer cells.

\section{Patients and methods \\ Patients}

The five patients described in this study were identified among 84 patients with suspected HNPCC who were referred to our Department for genetic counselling between June 1997 and September 2004. The five patients had a family history fulfilling at least one of the Bethesda guidelines ${ }^{15}$ and were selected because germline analysis had previously shown that they carried four MLH1 missense mutations of uncertain pathogenicity. The patients gave informed consent to genetic testing and to the additional studies required for this project. Each mutation was also searched in the germ line of a reference population panel of 155 healthy volunteers. Mutations previously identified as $M L H 1$ polymorphisms, missense changes occurring in patients harbouring chain-terminating mutations of $\mathrm{MSH} 2$ and $\mathrm{MLH} 1$ and mutation also found among the control subjects were not considered for this study.

\section{Sequence analysis of $\mathrm{MLH1}$ and $\mathrm{MSH} 2$}

Sequence analysis of $M L H 1$ and $M S H 2$ was carried out using lymphocyte DNA as previously described. ${ }^{16}$

\section{Immunohistochemistry of MLH1 and MSH2}

Immunohistochemical analysis of MLH1 and MSH2 protein expression was performed on paraffin-embedded tumour samples following antigen retrieval. ${ }^{16}$ The following antibodies and dilutions were used: anti-MLH1 protein: clone G168-728, 1:50 dilution (PharMingen, San Diego, CA, USA); anti-MSH2 protein: clone FE11, 1:100 dilution (Oncogene Research Products, Cambridge, MA, USA).

\section{Microsatellite instability analysis}

MSI was assessed by analysing the five recommended microsatellite markers included in the working reference panel: BAT25, BAT26, D2S123, D17S250 and D5S346. ${ }^{17}$ In addition, we studied a further five alternative loci also 
recommended for the assessment of MSI in HNPCC: BAT40, D18S58, D18S55, D18S61, MFD27. ${ }^{17}$ Microsatellite sequences were amplified from tumour and lymphocyte DNA, PCR products were analysed by high-resolution horizontal electrophoresis. ${ }^{16}$ Instability of the five markers included in the working reference panel was also assessed by automated allele sizing following capillary electrophoresis using the GeneScan 3.7 software (Applied Biosystems, Foster City, CA, USA). For this purpose microsatellite sequences were PCR amplified using oligonucleotide primer pairs in which the forward primer was $5^{\prime}$-fluorescence labelled with the following fluorochrome tags VIC (BAT26), NED (BAT25), FAM (D2S123), FAM (D17S250), PET (D5S346). PCR primers and PCR conditions are available from $\mathrm{E}$ Porfiri. The amplified products were analysed using an ABI 310 Genetic Analyzer (Applied Biosystems) using $1 \mu \mathrm{l}$ of the PCR product according to the manufacturer's recommendations.

\section{MLPA analysis}

MLPA was performed using the MRC-Holland (Amsterdam, Holland) HNPCC probe kit, according to the supplier's protocol. ${ }^{18}$ A $40-55 \%$ decrease of the area of an MSH2 or MLH1 exon peak compared to the wild-type control samples was considered as indicative of a heterozygous deletion of that exon.

\section{Coding mononucleotide repeats analysis}

We studied the occurrence of mutations within the sequence of the following five coding mononucleotide repeats: TGF $\beta R I I$ exon $3-\mathrm{A}_{10}$, MSH6 exon $3-\mathrm{C}_{8}, B A X$ exon $3-$ $\mathrm{G}_{8}$, PTEN exon $7-\mathrm{A}_{6}$, PTEN exon $8-\mathrm{A}_{6}$ and TP53 exon 11- $\mathrm{A}_{6}$. Repeat sequences were PCR amplified from tumour DNA and sequenced.

\section{Cloning of MLH1 and PMS2}

The entire open reading frame (ORF) of MLH1 and PMS2 was amplified using cDNA derived from 293 cells (PCR primers and PCR conditions are available from E Porfiri). PCR products were cloned into the pCAN expression vector, a modification pCDNA3 (Invitrogen, Carlsbad, CA, USA) containing the sequence of an 11 amino-acid myc-Tag which was placed at the amino terminus of MLH1 and PMS2 to generate pCAN-myc-MLH1 and pCAN-mycPMS2. MLH1 cDNA was also cloned in the pGEX4T-1 (Amersham Biosciences, Piscataway, NJ, USA) bacterial expression vector to generate the glutathione-s-transferase (GST)-MLH1 fusion gene GST-MLH1. The whole ORFs of MLH1 and of PMS2 were verified by sequencing.

\section{MLH1 site-directed mutagenesis}

Using a PCR-based site-direct mutagenesis method ${ }^{8}$ we generated four MLH1 variants each containing one of the missense mutations found in our patients: MLH1(P648S), MLH1(L559R), MLH1(K618A) and MLH1(Y646C). A further
MLH1 variant was generated by introducing the R659P (CGA $\rightarrow$ CCA) mutation, which is known to impair the interaction of the MLH1 protein with PMS2 in vitro. ${ }^{8,10}$ PCR products were cloned in pGEX4T-1 to obtain the following constructs pGEX-GST-MLH1(P648S), pGEX-GST-MLH1 (L559R), pGEX-GST-MLH1(K618A), pGEX-GST-MLH1(Y646C) and pGEX-GST-MLH1(R659P). Mutated versions of MLH1 were also cloned in the pCAN expression vector to obtain pCAN-myc-MLH1(P648S), pCAN-myc-MLH1(L559R), pCANmyc-MLH1(K618A), pCAN-myc-MLH1(Y646C). All mutations were confirmed by sequence analysis.

\section{Expression of MLH1 and PMS2 and HCT116 and 293 cells}

HCT-116 cells were transfected or cotransfected with the required vectors expressing wild-type or mutant MLH1 or wild-type PMS2 using LipofectAMIN ${ }^{\mathrm{TM}} 2000$ (Invitrogen) as specified by the manufacturer. Cells were harvested after $48 \mathrm{~h}$ and lysed in $20 \mathrm{mM}$ Tris- $\mathrm{HCl}, \mathrm{pH} 8,135 \mathrm{mM} \mathrm{NaCl}$, $1.5 \mathrm{mM} \mathrm{MgCl}_{2}, 1 \mathrm{~mm}$ EGTA, 1\% Triton X-100, 10\% glycerol, protease inhibitors. Equivalent amounts of cell protein $(50 \mu \mathrm{g})$ were analysed by sodium dodecyl sulphate-polyacrylamide gel electrophoresis (SDS-PAGE) and transferred to a PVDF membrane (Millipore, Billerica, MA, USA). The expression of myc-MLH1 and myc-PMS2 proteins was assessed by Western immunoblotting using a mouse monoclonal antibody to the myc-Tag (Clone 9E10, 1:600 dilution, Santa Cruz Biotechnology).

Human embryonic kidney 293 cells were transfected with pCAN-myc-PMS2 using LipofectAMIN ${ }^{\mathrm{TM}} 2000$ (Invitrogen) and the expression of myc-PMS2 protein was assessed by Western immunoblotting using an antibody to the myc-Tag (Clone 9E10, 1:600 dilution. Santa Cruz Biotechnology). Pooled 293 cells lysates were used as a source of wild-type PMS2 protein for the MLH1-PMS2 interaction assay as described in the following paragraph (Figure 1).

\section{MLH1-PMS2 in vitro interaction assay}

Vectors expressing wild-type GST-MLH1 or mutant GSTMLH1 were transformed into Escherichia coli. BL21 cells and proteins were purified using glutathione-agarose beads. ${ }^{10}$ Approximately $800 \mathrm{ng}$ of wild-type or mutant MLH1 proteins were prebound to glutathione-agarose beads and incubated for $2 \mathrm{~h}$ with $1.5 \mathrm{mg}$ of total cell protein from a lysate of 293 cells expressing myc-PMS2 in $500 \mu \mathrm{l}$ of binding buffer $(20 \mathrm{~mm}$ Tris- $\mathrm{HCl} \mathrm{pH} 7.5,10 \%$ glycerol, $150 \mathrm{~mm} \mathrm{NaCl}, 5 \mathrm{~mm}$ EDTA, $1 \mathrm{mM}$ dithiothreitol, $0.1 \%$ Tween 20 and protease inhibitors). Beads were washed three times and bound proteins were resolved by SDSPAGE. Proteins were transferred to a PVDF membrane (Millipore) and the assembly of the GST-MLH1/myc-PMS2 complex was detected by Western immunoblotting with a monoclonal antibody to the myc-tag (Clone 9E10, 1:600 dilution, Santa Cruz Biotechnology). In order to confirm 
a

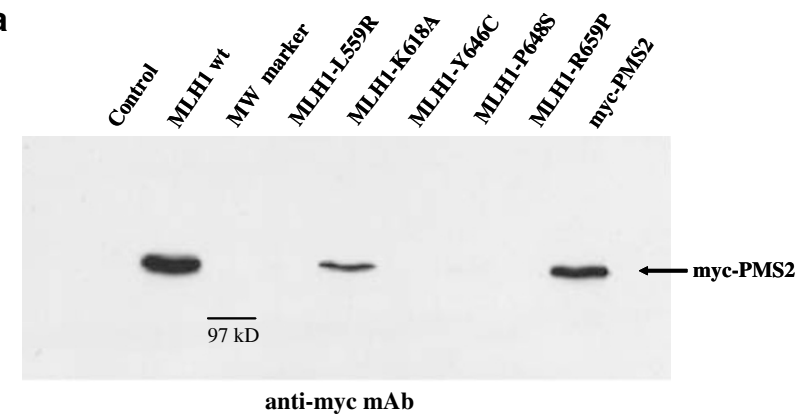

b

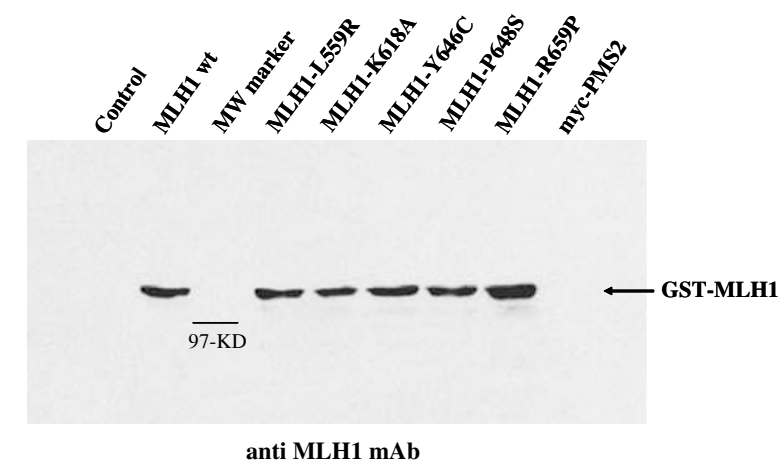

Figure 1 Interaction of wild-type MLH1 and of MLH1 missense variants with PMS2 in vitro. Purified GST-MLH1 proteins and GSTMLH1 missense variants were expressed in E. coli and $800 \mathrm{ng}$ of purified protein were prebound to glutathione-agarose beads and incubated with a total cell lysate of 293 cells expressing myc-PMS2. Bound proteins were resolved by SDS-PAGE and transferred to a PVDF membrane. Assembly of the GST-MLH1/myc-PMS2 complex was detected by Western immunoblotting with a monoclonal antibody to the myc-tag. Control: purified GST protein, MLH1 wt: GST-MLH1 wildtype, MLH1-L559R: GST-MLH1(L559R), MLH1-K618A: GSTMLH1(K618A), MLH1-Y646C: GST-MLH1(Y646C), MLH1-P648S: GST-MLH1(P648S), MLH1-R659P: GST-MLH1(R659P). myc-PMS2: $50 \mu \mathrm{g}$ of a lysate of 293 cell transfected with pCAN-myc-PMS2. (b) Amounts of wild-type or mutant GST-MLH1 protein used in the MLH1/ PMS2 interaction test. The PVDF membrane used to study the assembly of the MLH1/PMS2 complex (a) was probed with a monoclonal antibody to the MLH1 protein. that equal amounts of wild type or mutant GST-MLH1 proteins were used in the assay, the same PVDF membrane was subsequently stripped of the bound antibodies and probed with a monoclonal antibody to the MLH1 protein (Clone Ab-1, 1:150 dilution, Calbiochem Immunochemicals, Merck Biosciences, Darmstadt, Germany).

\section{Results}

\section{Patient characteristics and results of genetic studies}

We identified four germline MLH1 missense mutations in five unrelated patients who underwent genetic testing for suspected HNPCC (Table 1). The mutations were P648S $(\mathrm{CCC} \rightarrow$ TCC), L559R (CTG $\rightarrow$ CGG), K618A (AAG $\rightarrow$ GCG) and $\mathrm{Y} 646 \mathrm{C}(\mathrm{TAT} \rightarrow \mathrm{TGT})$. These genetic variants affected conserved amino-acid residues causing nonconservative amino-acid changes ${ }^{19}$ in addition we did not find them in a control group of 155 healthy individuals.

The P648S (CCC $\rightarrow$ TCC) mutation was found in a 63year-old patient with a mucinous adenocarcinoma of the right colon whose family history met the Amsterdam Criteria $^{1}$ as the patient's brother and father developed a tumour of the colon at the age of 42 and 56 years, respectively. In addition one son of the affected brother was diagnosed with an adenocarcinoma of the caecum at 36. The assessment of MSI showed instability of four of the 10 microsatellite markers BAT25, BAT26, BAT40 and D18S58. Immunohistochemistry showed loss of MLH1 protein expression whereas the expression of MSH2 was normal. MPLA did not show any abnormality of MLH1 and MSH2.

The mutation L559R (CTG $\rightarrow$ CGG) was found in a 63year-old patient who was diagnosed with a high-grade adenocarcinoma of the right colon and who had a familial history of colon and pancreatic cancer. MSI analysis showed instability of five microsatellite markers: D18S58, BAT25, BAT26, BAT40 and D17S250. Immunohistochemistry showed loss of MLH1 and normal MSH2 protein expression. MPLA did not show any abnormality of $M L H 1$ and $\mathrm{MSH} 2$.

Table 1 Clinical, genetic and functional features associated with the four MLH1 missense variants and their potential relationship with HNPCC

\begin{tabular}{|c|c|c|c|c|c|c|c|c|c|c|}
\hline $\begin{array}{l}\text { Patient } \\
\text { no. }\end{array}$ & $\begin{array}{l}\text { Family } \\
\text { history }\end{array}$ & Mutation ( $A A$ change) & Type of AA change & $M L P A$ & $\begin{array}{l}\text { MSI } \\
(\%)\end{array}$ & $\begin{array}{l}I H C \\
M L H 1\end{array}$ & $\begin{array}{l}\text { IHC } \\
\text { MSH2 }\end{array}$ & $\begin{array}{l}\text { Interaction } \\
\text { with PMS2 }\end{array}$ & $\begin{array}{l}\text { In vivo } \\
\text { expression }\end{array}$ & $\begin{array}{l}\text { Relationship } \\
\text { with HNPCC }\end{array}$ \\
\hline 277 & Amsterdam & $\mathrm{CCC} \rightarrow \mathrm{TCC}(\mathrm{P} 648 \mathrm{~S})$ & $\begin{array}{l}\text { Nonpolar to } \\
\text { polar }\end{array}$ & WT & 40 & Neg. & Pos. & No & No & Likely \\
\hline 230 & Bethesda & CTG $\rightarrow$ CGG (L559R) & $\begin{array}{l}\text { Nonpolar to } \\
\text { positive charged }\end{array}$ & WT & 50 & Neg. & Pos. & No & No & Probable \\
\hline 107 & Bethesda & $\mathrm{AAG} \rightarrow \mathrm{GCG}(\mathrm{K} 618 \mathrm{~A})$ & $\begin{array}{l}\text { Positive charged } \\
\text { to nonpolar }\end{array}$ & NA & 10 & Neg. & Pos. & Decreased & Decreased & Unlikely \\
\hline $\begin{array}{l}37 \\
417\end{array}$ & $\begin{array}{l}\text { Bethesda } \\
\text { Bethesda }\end{array}$ & TAT $\rightarrow$ TGT $($ Y646C) & Polar to nonpolar & $\begin{array}{l}\text { NA } \\
\text { ND }\end{array}$ & $\begin{array}{l}10 \\
40\end{array}$ & $\begin{array}{l}\text { Neg. } \\
\text { Neg. }\end{array}$ & $\begin{array}{l}\text { Pos. } \\
\text { Pos. }\end{array}$ & No & Yes & Unlikely \\
\hline
\end{tabular}

Amsterdam, patients fulfilling the Amsterdam Criteria; Bethesda, patients fulfilling at least one of the Betesda Guidelines; MLPA, multiplex ligationdependent probe amplification; WT, wild-type; NA, DNA sample not available; ND, not diagnostic; MSI, percentage of the microsatellite markers showing instability; IHC, immunohistochemistry; In vivo expression, expression of MLH1 mutants in HCT116 cells; Neg., negative; Pos., positive. 
The mutation K618A (AAG $\rightarrow$ GCG) was found in a patient who developed an adenocarcinoma of the rectum at the age of 39 years without any significant family history of HNPCC-related tumours. ${ }^{16}$ Immunohistochemistry showed loss of MLH1 protein but normal MSH2 expression whereas microsatellite analysis demonstrated instability of BAT 40 only.

The mutation Y646C (TAT $\rightarrow$ TGT) was found in two patients, the first patient was a 72-year-old woman who developed breast cancer at the age of 48 years and rectal cancer at 72 years. She had a family history of colon cancer and early-onset brain cancer. ${ }^{16}$ Of the 10 microsatellite markers analysed only D18S55 showed instability. Immunohistochemistry demonstrated loss of MLH1 protein expression in the tumour tissue but normal expression of MSH2. The same mutation was found in an unrelated patient who was diagnosed with an adenocarcinoma of the right colon at the age of 51 years and had a familial history of colon cancer and brain cancer. MSI analysis showed instability of BAT 26 and BAT 40, D17S250 and D5S346. Immunohistochemistry showed loss of MLH1 protein and normal expression of MSH2. MPLA gave inconclusive results perhaps due to the poor quality of the DNA. Unfortunately we were unable to carry out segregation analysis in any of the five families because in some cases the individuals involved declined testing and because paraffin-embedded tissue samples from affected family members who had died were often not available.

\section{Coding mononucleotide repeats analysis}

Impairment of the DNA MMR system results in the accumulation of replication errors which may affect mononucleotide repeats situated within the coding sequence of regulatory genes. Nevertheless none of the five patients showed somatic abnormalities of the six coding repeats we studied TGF $\beta R I I$ exon $3-\mathrm{A}_{10}$, MSH6 exon $3-\mathrm{C}_{8}$, $B A X$ exon3- $\mathrm{G}_{8}$, PTEN exon $7-\mathrm{A}_{6}$, PTEN exon8- $\mathrm{A}_{6}$ and TP53 exon11-A 6 .

\section{In vitro interaction of MLH1 variants with PMS2}

The four missense mutations we found occurred within the carboxy-terminal domain of the MLH1 protein which is critical for its interaction with PMS2. ${ }^{10}$ Therefore, we studied if these mutations impaired the binding of MLH1 to PMS2 using an in vitro interaction assay in which wildtype or mutant GST-MLH1 was prebound to glutathione agarose beads and added to a lysate of 293 cells-expressing wild-type myc-PMS2. Beads were recovered and the bound proteins were analysed by Western immunoblotting using an anti-myc monoclonal antibody. These experiments showed that the MLH1 mutations P648S, L559R and Y646C disrupted the interaction of GST-MLH1 with mycPMS2 in vitro (Figure 1a). The MLH1 (K618A) mutant showed a reduced ability to bind PMS2 (Figure 1a). As expected wild-type MLH1 was able to interact with PMS2, whereas the R659P mutation was sufficient to abolish such interaction as previously described ${ }^{8,10}$ (Figure 1a). We ascertained that an equivalent amount of each GSTMLH1 mutant was used in each interaction test, by reanalysing the immunoblot membranes with an antiMLH1 antibody (Figure 1b).

\section{Expression of MLH1 and PMS2 protein in HCT-116 human colon carcinoma cells}

The level of expression of wild-type or mutant MLH1 was studied following the transfection of the appropriate vectors into HCT-116 colon carcinoma cells which do not express endogenous MLH1 and PMS2 protein. ${ }^{20}$ When we transfected pCAN-myc-MLH1 alone we detected a very low MLH1 protein expression and no PMS2 expression (data not shown), this was probably due to the fact that a sufficient level of PMS2 could not be stabilized during the transient transfection. ${ }^{12}$ Consistent with previous observations, the levels of ectopically expressed myc-MLH1 and myc-PMS2 were significantly increased when we cotransfected HCT116 cells with pCAN-myc-MLH1 and pCANmyc-PMS2, $^{12}$ therefore the expression of each MLH1 missense variant was studied following cotransfection with wild-type PMS2. We did not detect any myc-MLH1 or mycPMS2 protein expression when we cotransfected pCANmyc-PMS2 with pCAN-myc-MLH1(P648S) or pCAN-mycMLH1(L559R), suggesting that these MLH1 mutant proteins were unstable and/or unable to bind and stabilize PMS2 in vivo (Figure 2). Cotransfection of pCAN-myc-PMS2 and pCAN-myc-MLH1(K618A) resulted in a slightly decreased level of myc-MLH1 protein expression whereas cotransfection with pCAN-myc-MLH1(Y646C) resulted in a level of myc-MLH1 protein expression similar to that observed when using wild type MLH1 (Figure 2).

\section{Discussion}

Counselling patients with suspected HNPCC, who harbour missense mutations of MMR genes, may prove challenging, given the often uncertain pathogenicity of these abnormalities and the difficulty to estimate the cancer risk of the mutation carrier. As segregation analysis is seldom feasible, the role of missense mutations is often inferred from the patient's familial cancer history and occurrence of MSI, ${ }^{7,21,22}$ or from the results of assays testing the functional impairment caused by the mutation to the MMR system. ${ }^{8,10-13}$ We hypothesized that a more reliable pathogenicity assessment could be achieved by studying the genetic and functional characteristics of the mutations as well as their relationship with other features which are often indicative of HNPCC such as family history, occurrence of MSI, MMR gene expression abnormalities. The four missense mutations we studied shared significant genetic and pathological similarities: they affected conserved amino-acid residues, caused nonconservative 


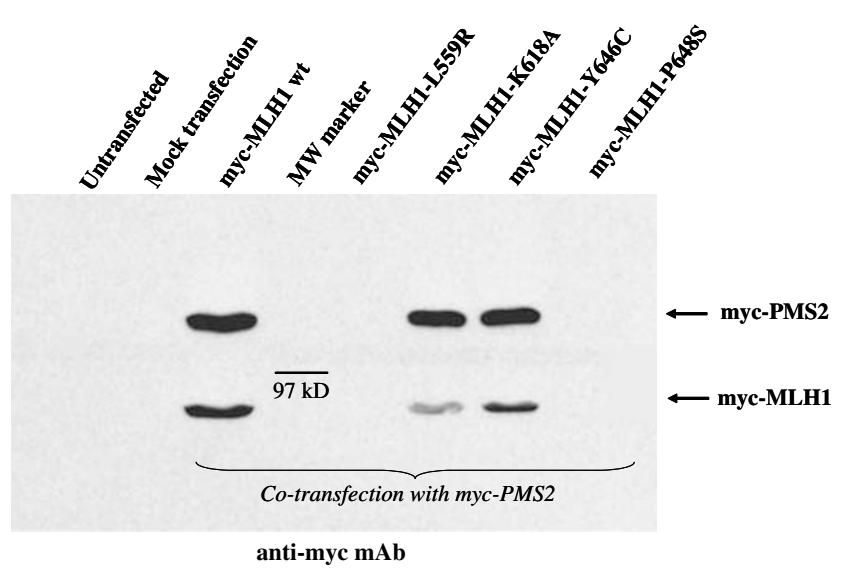

Figure 2 Expression of wild-type or mutant MLH1 and PMS2 in HCT116 cells. HCT116 cells were cotransfected with pCAN-myc-PMS2 and with pCAN-myc-MLH1 or with pCAN-myc-MLH1(L559R), pCANmyc-MLH1(K618A), pCAN-myc-MLH1(Y646C), pCAN-mycMLH1(P648S) as indicated. Following transfection HCT116 cells were lysed and equivalent amounts of cell protein were analysed by Western immunoblotting using a monoclonal antibody to the myc-tag to detect the expression of myc-MLH1 and myc-PMS2. Control: untransfected cells. Mock transfection: cells transfected with empty vector.

amino-acid changes and occurred within the domain of interaction of MLH1 with PMS2, ${ }^{10}$ in addition we did not find them in a control panel of 155 healthy individuals and were associated with loss of MLH1 expression in the tumour tissue. These features were typical of deleterious mutations and suggested that all four missense variants were potentially pathogenic nevertheless significant differences emerged from the study of the clinical and functional characteristics of these genetic abnormalities.

It is likely that the P648S mutation had a causative role in HNPCC as it was found in a patient with a strong family history of colon cancer who developed a high-grade mucinous tumour showing high MSI. In addition this mutation was able to disrupt the assembly of the MutL $\alpha$ complex in vitro and abolished MLH1 expression in HCT116 cells. Consistent with our views, the P648S mutation was previously shown to affect the stability of the MLH1 protein in vivo and was found to segregate with the disease in a large HNPCC family. ${ }^{23}$ However, it has also been suggested that this mutation could represent an MLH1 polymorphism as it was detected in three healthy individuals from a reference panel of 50 controls subjects. ${ }^{6}$ It is difficult to explain the discrepancy between this observation and our data. It is possible, although unlikely, that genetic differences existed between our reference population and the population studied by Cravo et al nevertheless if this variant had such a high frequency then it would perhaps have been reported by other authors. In addition, MPLA did not show other germ line abnormalities of MLH1 and MSH2 which could be responsible of the strong HNPCC phenotype of this patient, therefore we believe that the MLH1 P648S mutation is pathogenic and that families who harbour this abnormality should be counselled accordingly.

The analysis of the clinical and functional characteristics of the L559R mutation also suggested that this genetic abnormality was probably linked to HNPCC. This mutation was found in a patient with a high-grade, right-sided tumour which showed high MSI, but with a weak family history of colon cancer. Nevertheless the mutation was able to abolish the binding of MLH1 to PMS2 in vitro, impaired MLH1 expression in HCT116 cells and was previously described in a patient from a family with suspected HNPCC. ${ }^{24}$ Disease causing mutations have been found in patients with atypical family history ${ }^{25}$ suggesting that the family phenotype, although important, should not be used as the sole criterion to establish the pathogenicity of missense variants. In these patients, the presence of other clinicopathological features commonly associated with HNPCC and the results of functional studies should be taken into account when assessing the biological significance of unclassified MMR gene variants.

The pathogenicity of the remaining 2 MLH1 missense mutations, K618A and Y646C, was uncertain. We found the K618A mutation in a patient with early-onset rectal cancer but without any significant family history. ${ }^{16}$ The mutation was associated with low microsatellite instability and impaired only in part the assembly of the MutL $\alpha$ complex and the expression of MLH1 in HCT116 cells. These observations and the fact that this abnormality was previously described in a healthy control ${ }^{26}$ suggest that it represents an MLH1 polymorphism rather than a disease causing mutation. On the other hand, this genetic abnormality is one of the most common alterations found in HNPCC families. ${ }^{2}$ Such contradictory evidence is an example of the dilemma often posed by the interpretation of missense mutations. However, recently published data ${ }^{27}$ also suggest that the K618A mutation is not pathogenic therefore we believe that individuals harbouring this abnormality should be reassured and further genetic tests, for example MLPA, should be carried out in families with a strong phenotype.

The Y646C mutation was probably not linked to HNPCC. We found it in two unrelated patients showing a weak family history. The mutation was associated with loss of MLH1 expression in the tumour tissue in both cases nevertheless, while one patient displayed a high MSI, the other showed instability of only one microsatellite marker. The functional analysis of MLH1(Y646C) was inconclusive as the mutation was able to disrupt the interaction between the MLH1 and PMS2 proteins in vitro but did not affect the ectopic expression of myc-MLH1(Y646C) and myc-PMS2 in HCT 116 cells. These observations would suggest that MLH1(Y646C) was able to stabilize PMS2 through a mechanism independent from the formation of the MLH1-PMS2 heterodimer, thus contradicting the current 
views regarding the biochemistry of MLH1-regulated PMS2 post-translational stabilization. ${ }^{12,20}$ The conflicting results of the functional assays, the different levels of MSI found in the two patients as well as the weak family phenotype indicated that the Y646C mutation probably represents a rare $M L H 1$ polymorphism. MPLA either gave inconclusive results or was not feasible in the two patients harbouring the Y646C mutation, nevertheless it could be argued that given the weak family history it is unlikely that these patients carried large genomic alterations of MLH1 or MSH2 which are usually associated with a stronger phenotype. Consistent with our views, recently published data also indicate that this mutation is not pathogenic. ${ }^{27}$

In conclusion, although none of the mutation features we analysed can be considered individually sufficient to predict the biological role of $M L H 1$ missense variants, our data suggest that a plausible pathogenicity assessment can be reached by studying the correlation existing between the clinical, genetic and functional characteristics of these genetic abnormalities. Among them, occurrence of high MSI, disruption of MutL $\alpha$ complex assembly, loss of in vivo expression and, to some extent, patient family history were those more indicative of a correlation with HNPCC, whereas the evolutionary conservation of the mutated amino acid, the type of amino-acid change, the frequency of the mutation and loss of MLH1 protein expression in the tumour, which could be due to a number of somatic or epigenetic causes, seemed to have a secondary role. Given the genetic heterogeneity of HNPCC and the frequency of missense mutations, more research is necessary to better understand the biological role of these genetic abnormalities. Areas which warrant further study are the development of validated and replicable assays to test the function of MMR gene mutants as well as the design of cooperative clinical trials studying the cancer risk of individuals carrying unclassified variants of MMR genes.

\section{References}

1 Lynch HT, de la Chapelle A: Hereditary colorectal cancer. $N$ Engl J Med 2003; 348: 919-932.

2 Peltomaki P, Vasen $\mathrm{H}$ : Mutations associated with HNPCC predisposition - Update of ICG-HNPCC/INSiGHT mutation database. Dis Markers 2004; 20: 269-276.

3 Peltomaki P: Role of DNA mismatch repair defects in the pathogenesis of human cancer. J Clin Oncol 2003; 21: 1174-1179.

4 Guerrette S, Wilson T, Gradia S, Fishel R: Interactions of human hMSH2 with hMSH3 and hMSH2 with hMSH6: examination of mutations found in hereditary nonpolyposis colorectal cancer. Mol Cell Biol 1998; 18: 6616-6623.

5 Li GM, Modrich P: Restoration of mismatch repair to nuclear extracts of H6 colorectal tumor cells by a heterodimer of human MutL homologs. Proc Natl Acad Sci USA 1995; 92: 1950-1954.

6 Cravo M, Afonso AJ, Lage P et al: Pathogenicity of missense and splice site mutations in $h M S H 2$ and $h M L H 1$ mismatch repair genes: implications for genetic testing. Gut 2002; 50: 405-412.

7 Genuardi M, Carrara S, Anti M, Ponz de Leon M, Viel A: Assessment of pathogenicity criteria for constitutional missense mutations of the hereditary nonpolyposis colorectal cancer genes MLH1 and MSH2. Eur J Hum Genet 1999; 7: 778-782.
8 Nystrom-Lahti M, Perrera C, Raschle M et al: Functional analysis of MLH1 mutations linked to hereditary nonpolyposis colon cancer. Genes Chromosomes Cancer 2002; 33: 160-167.

9 Yuen ST, Chan TL, Ho JW et al: Germline, somatic and epigenetic events underlying mismatch repair deficiency in colorectal and HNPCC-related cancers. Oncogene 2002; 21: 7585-7592.

10 Guerrette S, Acharya S, Fishel R: The interaction of the human MutL homologues in hereditary nonpolyposis colon cancer. J Biol Chem 1999; 274: 6336-6341.

11 Kondo E, Suzuki H, Horii A, Fukushige S: A yeast two-hybrid assay provides a simple way to evaluate the vast majority of $h M L H 1$ germ-line mutations. Cancer Res 2003; 63: 3302-3308.

12 Trojan J, Zeuzem S, Randolph A et al: Functional analysis of $h M L H 1$ variants and HNPCC-related mutations using a human expression system. Gastroenterology 2002; 122: 211-219.

13 Ellison AR, Lofing J, Bitter GA: Functional analysis of human MLH1 and MSH2 missense variants and hybrid human-yeast MLH1 proteins in Saccharomyces cerevisiae. Hum Mol Genet 2001; 10: $1889-1900$

14 Vasen HF, Wijnen JT, Menko FH et al: Cancer risk in families with hereditary nonpolyposis colorectal cancer diagnosed by mutation analysis. Gastroenterology 1996; 110: 1020-1027.

15 Rodriguez-Bigas MA, Boland CR, Hamilton SR et al: A National Cancer Institute Workshop on Hereditary Nonpolyposis Colorectal Cancer Syndrome: meeting highlights and Bethesda guidelines. J Natl Cancer Inst 1997; 89: 1758-1762.

16 Scartozzi M, Bianchi F, Rosati S et al: Mutations of $h M L H 1$ and $h M S H 2$ in patients with suspected hereditary nonpolyposis colorectal cancer: correlation with microsatellite instability and abnormalities of mismatch repair protein expression. J Clin Oncol 2002; 20: 1203-1208.

17 Boland CR, Thibodeau SN, Hamilton SR et al: A National Cancer Institute Workshop on Microsatellite Instability for cancer detection and familial predisposition: development of international criteria for the determination of microsatellite instability in colorectal cancer. Cancer Res 1998; 58: 5248-5257.

18 Pastrello C, Baglioni S, Tibiletti MG et al: Stability of BAT26 in tumours of hereditary nonpolyposis colorectal cancer patients with MSH2 intragenic deletion. Eur J Hum Genet 2006; 14: $63-68$.

$19 \mathrm{Ng}$ PC, Henikoff S: SIFT: predicting amino acid changes that affect protein function. Nucl Acids Res 2003; 31: 3812-3814.

20 Chang DK, Ricciardiello L, Goel A, Chang CL, Boland CR: Steady-state regulation of the human DNA mismatch repair system. J Biol Chem 2000; 275: 18424-18431.

21 Liu B, Parsons R, Papadopoulos N et al: Analysis of mismatch repair genes in hereditary non-polyposis colorectal cancer patients. Nat Med 1996; 2: 169-174.

22 Bisgaard ML, Jager AC, Myrhoj T, Bernstein I, Nielsen FC: Hereditary non-polyposis colorectal cancer (HNPCC): phenotype-genotype correlation between patients with and without identified mutation. Hum Mutat 2002; 20: 20-27.

23 Raevaara TE, Gerdes AM, Lonnqvist KE et al: HNPCC mutation MLH1 P648S makes the functional protein unstable, and homozygosity predisposes to mild neurofibromatosis type 1 . Genes Chromosomes Cancer 2004; 40: 261-265.

24 Taylor CF, Charlton RS, Burn J, Sheridan E, Taylor GR: Genomic deletions in MSH2 or MLH1 are a frequent cause of hereditary non-polyposis colorectal cancer: identification of novel and recurrent deletions by MLPA. Hum Mutat 2003; 22: 428-433.

25 Wijnen J, Khan PM, Vasen $\mathrm{H}$ et al: Hereditary nonpolyposis colorectal cancer families not complying with the Amsterdam criteria show extremely low frequency of mismatch-repair-gene mutations. Am J Hum Genet 1997; 61: 329-335.

26 Samowitz WS, Curtin K, Lin HH et al: The colon cancer burden of genetically defined hereditary nonpolyposis colon cancer. Gastroenterology 2001; 121: 830-838.

27 Raevaara TE, Korhonen MK, Lohi $\mathrm{H}$ et al: Functional significance and clinical phenotype of nontruncating mismatch repair variants of MLH1. Gastroenterology 2005; 129: 537-549. 